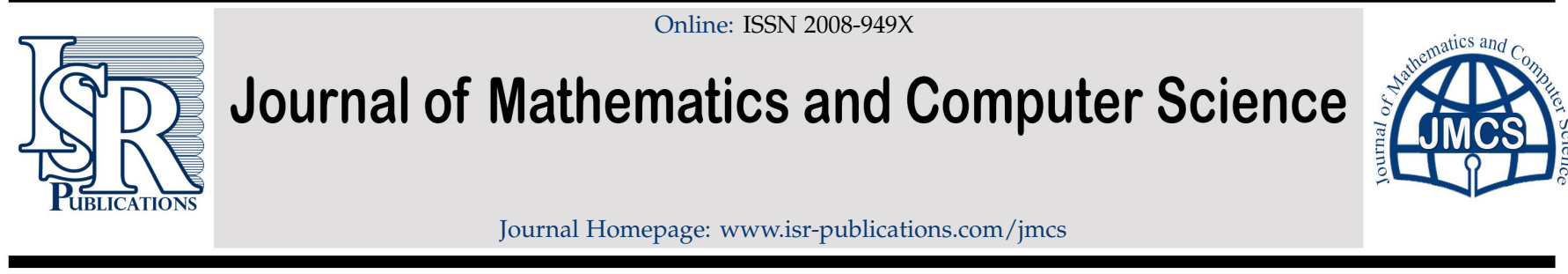

\title{
On some characterization of Q-complex fuzzy sub-rings
}

\author{
Muhammad Gulzar ${ }^{a, *}$, Dilshad Alghazzawi ${ }^{\text {b }}$, M. Haris Mateen ${ }^{\mathrm{c}}$, M. Premkumard,e \\ ${ }^{a}$ Department of Mathematics, Government College University Faisalabad, 38000, Pakistan. \\ ${ }^{b}$ Department of Mathematics, King Abdulaziz University (Rabigh), Saudi Arabia. \\ ${ }^{c}$ Department of Mathematics, University of the Punjab, Lahore, Pakistan. \\ ${ }^{d}$ Research Scholar, PG and Research Department of Mathematics, Jamal Mohamed College (Autonomous), (Affiliated to \\ Bharathidasan University), India. \\ e Department of Mathematics, Kongunadu College of Engineering and Technology, Tiruchirappalli-620020, Tamilnadu, India.
}

\begin{abstract}
In this paper, we introduce the idea of Q-complex fuzzy sub-ring (Q-CFSR) and discuss its various algebraic aspects. We prove that every Q-CFSR generates two Q-fuzzy sub-rings (Q-FSRs). We also present the concept of level subsets of Q-CFSR and show that level subset of Q-CFSR form sub-ring. Furthermore, we extend this idea to define the notion of the direct product of two Q-CFSR Moreover, we investigate the homomorphic image and inverse image of Q-CFSR.
\end{abstract}

Keywords: Complex fuzzy set (CFS), Q-complex fuzzy set (Q-CFS), Q-complex fuzzy sub-ring (Q-CFSR).

2020 MSC: 03E72, 08A72, $20 \mathrm{~N} 25$.

(C)2021 All rights reserved.

\section{Introduction}

Rings were first systematized as a extension of Dedekind domains that appear in polynomial rings, invariant rings, and number theory. Recently, ring theory is the most important branch of the mathematics that has been broadly used in cryptography, theoretical physics and algebraic geometry.

The fuzzy set theory is founded on the doctrine of concerning relative graded membership basing human mechanism of cognition as well as perception. Zahed [32] published his maiden well acknowledged research paper about fuzzy sets in 1965. Fuzzy logic overcame the data resulting from computational presumption that is obscure, inaccurate and sometimes biased also. Fuzzy logic provides the opportunity of including ambiguous human evaluation in calculating the problems. This theory also leads us to search for better way to resolve our daily life issues by proper technique of decision making. Many mathematician have applied various hybrid models of fuzzy sets and intuitionistic fuzzy sets to several algebraic structure such as non-associative ring [9, 10], time series [2, 24] and decision making [16]. Rosenfeld [20] initiated the concept of fuzzy subgroups and developed basic properties of fuzzy subgroups. For

\footnotetext{
*Corresponding author

Email addresses: 98kohly@gmail.com (Muhammad Gulzar), dalghazzawi@kau.edu.sa (Dilshad Alghazzawi),

harism.math@gmail.com (M. Haris Mateen), mprem.maths3033@gmail .com (M. Premkumar)
}

doi: $10.22436 /$ jmcs.022.03.08

Received: 2020-06-19 Revised: 2020-07-06 Accepted: 2020-07-21 
more development about fuzzy subgroup one can refer [21, 22]. Later on, Liu [13] introduced the concept of fuzzy invariant subgroups and fuzzy ideals in 1982. Malik and Mordeson [15] commenced the idea of fuzzy homomorphism of rings in 1992. Gang and Yun [5] explained the notion of fuzzy factor rings in 1998. Ramot et al [17, 18] started the conception of complex fuzzy sets in 2002. The enlargement of fuzzy sets to complex fuzzy sets is comparable to the extension of real numbers to complex numbers. This idea becomes more effective for researcher and quite different from fuzzy complex numbers innovated by Buckley [4]. In 2009, the various operations of complex fuzzy sets were founded by Zhang et al [33]. Yetkin and Olgun [26] studied the direct product of fuzzy subgroup and fuzzy sub-ring in 2011. Solairaju and Nagarajan [30] developed a new structure of Q-fuzzy subgroup groups and discussed some elementary properties of these groups. Lee et al [12] innovated the concept of fuzzy completely prime ideal. Thirunavukarasu et al. [27] illustrated a possible application including complex fuzzy representation of solar activity, forecasting problems, time series, signal processing application and compare the two national economies by using the concept of complex fuzzy relation. Azam et al [3] explored some properties of anti-fuzzy ideal in 2013. Selvachandran et al [23] developed a relationship between complex vague soft sets and investigated important human experience events in Malaysian and Chinese economies. Singh et al [25] portrayed the fixed point theorem by using the conception of complex fuzzy sets. In 2016, Thirunavukarasu et al [24] discussed a novel approach on energy of a complex fuzzy graph. Makamba and Murali [14] studied parabolic fuzzy subgroups in 2017. Kellil [11] defined the product of fuzzy sub-rings in 2018. Addis [1] explored the structure of fuzzy homomorphism on fuzzy subgroups. Hu et al. $[7,8]$ investigated the many distance measurements of complex fuzzy sets and the approximate comparisons between complex fuzzy sets based on the transition complex valued membership. The concept of Q-fuzzy sub-ring with respect to t-norm was introduced by Rasuli [19]. Many interesting results of $(\alpha, \beta)$-complex fuzzy hyper-ideal were presented by [6] in 2019. Trevijano et al. [29] investigated some characterization of annihilator on fuzzy subgroups. Yun and Xin [31] invented the complex sub-algebra by using the notion of complex fuzzy set and generalized this concept in BCK/BCI-algebra. We will extend the discussion of complex fuzzy sets (CFSs) by proposing a new concept of Q-CFSs by adding a second dimension in membership function of Q-fuzzy sets (Q-FSs). Complete characterization of many ring theory problems can be handled by Q-CFSs. This theory will be useful for mathematician in future research work. This paper is organized as follows. Section 2 contains the introductory definition of Qfuzzy sub-rings (Q-FSRs) and related result which play a key role for our further discussion. In Section 3 we define $\pi$-Q-complex fuzzy sub-rings ( $\pi$-Q-FSR), Q-CFSR and also prove that level subset of Q-CFSR is sub-ring of ring $S$, and vice versa. In Section 4 , we prove that the product of two Q-CFSRs is Q-CFSR and develop some results of the product of two Q-CFSRs. The image and inverse image of Q-CFSR under ring homomorphism is elucidated in Section 5.

\section{Preliminaries}

In this section, we define some basic definition of Q-FSs and CFSs, which will be used in our further discussion.

Definition 2.1 ([32]). A fuzzy set $A$ of a nonempty set $P$ is a mapping $A: P \rightarrow[0,1]$.

Definition 2.2 ([13]). A fuzzy set $A$ of a ring $S$ is called a fuzzy sub-ring(FSR) of $S$ if

1. $A(m-n) \geqslant \min \{A(m), A(n)\}, \forall m, n \in S ;$

2. $A(m n) \geqslant \min \{A(m), A(n)\}, \forall m, n \in S$.

Definition 2.3 ([26]). Let $Q$ and $S$ be any two sets. Then the mapping $A: S \times Q \rightarrow[0,1]$ is called a Q-fuzzy set (Q-FS) in S.

Definition 2.4 ([19]). A Q-FS A of ring $S$ is said to be Q-FSR if the following properties hold,

1. $A(m-n, q) \geqslant \min \{A(m, q), A(n, q)\}$, for all $m, n \in S$ and $q \in Q$; 
2. $A(m n, q) \geqslant \min \{A(m, q), A(n, q)\}$, for all $m, n \in S$ and $q \in Q$.

Definition $2.5([18])$. A CFS A of universe of discourse $P$ is identify with the membership function $\theta_{A}(m)=\eta_{A}(m) e^{i \varphi_{A}(m)}$ and is defined as

$$
\theta_{\mathrm{A}}: \mathrm{P} \rightarrow\{z \in \mathrm{C}:|z| \leqslant 1\} .
$$

This membership function receive all membership value from the unit disc on plane, where $i=\sqrt{-1}$, both $\eta_{A}(m)$ and $\varphi_{A}(m)$ are real valued such that $\eta_{A}(m) \in[0,1]$ and $\varphi_{A}(m) \in[0,2 \pi]$.

Definition 2.6 ([33]). Let A and B two CFSs of set P. The Cartesian product of CFSs A and B is defined as

$$
\theta_{A \times B}(m, n)=\eta_{A \times B}(m, n) e^{i \varphi_{A \times B}(m, n)}=\min \left\{\eta_{A}(m), \eta_{B}(n)\right\} e^{i \min \left\{\varphi_{A}(m), \varphi_{B}(n)\right\}} .
$$

Definition 2.7 ([19]). Let the mapping $f: S_{1} \rightarrow S_{2}$ be a homomorphism. Let $A$ and B be two Q-FSRs of $S_{1}$ and $S_{2}$, respectively, then $f(A)$ and $f^{-1}(B)$ are image of $A$ and the inverse image of $B$, respectively, defined as

1. $f(A)(n, q)=\left\{\begin{array}{ll}\sup \left\{A(m, q): m \in f^{-1}(n)\right\}, & \text { if } f^{-1}(n) \neq \emptyset, \\ 0, & \text { if } f^{-1}(n)=\emptyset,\end{array}\right.$ for every $n \in S_{2}$ and $q \in Q$;

2. $f^{-1}(B)(m, q)=B(f(m), q)$, for every $m \in S_{1}$ and $q \in Q$.

\section{Properties of Q-complex fuzzy sub-rings}

This section is devoted to the study of Q-CFS and Q-CFSR. We also found that a Q-CFSR generates two Q-FSRs. We also show that level subset of Q-CFSR form sub-ring of ring and investigate some fundamental properties of this ring.

Definition 3.1. A Q-CFS A of universe of discourse $P$ is represented by the membership function $\theta_{A}(m, q)=$ $\eta_{A}(m, q) e^{i \varphi_{A}(m, q)}$ and described as

$$
\theta_{\mathrm{A}}: \mathrm{P} \times \mathrm{Q} \rightarrow\{z \in \mathrm{C}:|z| \leqslant 1\} .
$$

This membership function receives all membership value from the unit disc on plane, where $i=\sqrt{-1}$, both $\eta_{A}(m, q)$ and $\varphi_{A}(m, q)$ are real valued such that $\eta_{A}(m, q) \in[0,1]$ and $\varphi_{A}(m, q) \in[0,2 \pi]$.

Throughout this paper we take membership function of Q-CFSs $A$ and $B$ such as $\theta_{A}(m, q)=\eta_{A}(m, q) e^{i \varphi_{A}(m, q)}$ and $\theta_{B}(m, q)=\eta_{B}(m, q) e^{i \varphi_{B}(m, q)}$.

Definition 3.2. A $\pi$-Q-fuzzy set ( $\pi$-Q-fuzzy set) $A_{\pi}$ of ring $S$ is called $\pi$-Q-FSR of $S$ if the following axioms hold:

1. $\eta_{A_{\pi}}((m-n), q) \geqslant \min \left\{\eta_{A_{\pi}}(m, q), \eta_{A_{\pi}}(n, q)\right\}$, for all $m, n \in S$ and $q \in Q$;

2. $\eta_{A_{\pi}}(m n, q) \geqslant \min \left\{\eta_{A_{\pi}}(m, q), \eta_{A_{\pi}}(n, q)\right.$, $\}$, for all $m, n \in S$ and $q \in Q$.

Theorem 3.3. Let $\mathrm{A}$ be $\pi-\mathrm{Q}-F S$ of $\mathrm{S}$. Then $\mathrm{A}$ is $\pi-\mathrm{Q}-F S R$ if and only if $\mathrm{A}$ is Q-FSR.

Definition 3.4. Let $A$ and $B$ be two Q-CFS of $S$. Then

1. a Q-CFS $A$ is homogeneous Q-CFS, if for all $m, n \in S$ and $q \in Q$, we have $\mu_{A}(m, q) \leqslant \mu_{A}(n, q)$ if and only if $\varphi_{A}(m, q) \leqslant \varphi_{A}(n, q)$;

2. a CFS $A$ is homogeneous CFS with $B$, if for all $m, n \in S$ and $q \in Q$, we have $\mu_{A}(m, q) \leqslant \mu_{B}(m, q)$ if and only if $\varphi_{A}(m, q) \leqslant \varphi_{B}(m, q)$.

In this paper, we shall use Q-CFS as homogeneous Q-CFS. 
Definition 3.5. A Q-CFS A of ring $S$ is called Q-CFSR of $S$ if

1. $\theta_{A}((m-n), q) \geqslant\left\{\theta_{A}(m, q), \theta_{A}(n, q)\right\}$, for all $m, n \in S$ and $q \in Q$;

2. $\theta_{A}(m n, q) \geqslant\left\{\theta_{A}(m, q), \theta_{A}(n, q),\right\}$, for all $m, n \in S$ and $q \in Q$.

The following result indicates that every Q-CFSR generates two Q-CFSRs.

Theorem 3.6. Let $\mathrm{A}=\left\{\mathrm{a}, \theta_{\mathrm{A}}(\mathrm{a}, \mathrm{q}): \mathrm{a} \in \mathrm{S}\right.$ be a $\mathrm{Q}-C F S$ of ring $\mathrm{S}$. Then $\mathrm{A}$ is a $\mathrm{Q}-C F S R$ of $\mathrm{S}$ iff:

1. the fuzzy set $\dot{A}=\left\{\left(a, \eta_{A}(a, q)\right): a \in S, \eta_{A}(a, q) \in[0,1], q \in Q\right\}$ is a Q-FSR;

2. the $\pi$-fuzzy set $\ddot{A}=\left\{\left(a, \varphi \_A(a, q)\right): m \in S, \varphi \_A(a, q) \in[0,2 \pi], q \in Q\right\}$ is a $\pi$-Q-FSR.

Proof. Suppose that $A$ be $Q-C F S R$ and $a, b \in S$ and $q \in Q$, then we have

$$
\begin{aligned}
\eta_{A}((a-b), q) e^{i \varphi_{A}((a-b), q)} & =\theta_{A}((a-b), q) \\
& \geqslant\left\{\theta_{A}(a, q), \theta_{A}(b, q)\right\} \\
& =\min \left\{\eta_{A}(a, q) e^{i \varphi_{A}(a, q)}, \eta_{A}(b, q) e^{i \varphi_{A}(b, q)}\right\} \\
& =\min \left\{\eta_{A}(a, q), \eta_{A}(b, q)\right\} e^{i \min \left\{\varphi_{A}(a, q), \varphi_{A}(b, q)\right\}}
\end{aligned}
$$

As $A$ is homogeneous, $\eta_{A}((a-b), q) \geqslant \min \left\{\eta_{A}(a, q), \eta_{A}(b, q)\right\}$ and $\varphi_{A}((a-b), q) \geqslant\left\{\varphi_{A}(a, q), \varphi_{A}(b, q)\right\}$. Moreover,

$$
\begin{aligned}
\eta_{A}(a b, q) e^{i \varphi_{A}(a b, q)} & =\theta_{A}(a b, q) \\
& \geqslant \min \left\{\theta_{A}(a, q), \theta_{A}(b, q)\right\} \\
& =\min \left\{\eta_{A}(a, q) e^{i \varphi_{A}(a, q)}, \eta_{A}(b, q) e^{i \varphi_{A}(b, q)}\right\} \\
& =\min \left\{\eta_{A}(a, q), \eta_{A}(b, q)\right\} e^{i \min \left\{\varphi_{A}(a, q), \varphi_{A}(b, q)\right\}} .
\end{aligned}
$$

As $A$ is homogeneous, $\eta_{A}(a b, q) \geqslant \min \left\{\eta_{A}(a, q), \eta_{A}(b, q)\right\}$ and $\varphi_{A}(a b, q) \geqslant\left\{\varphi_{A}(a, q), \varphi_{A}(b, q)\right\}$. Conversely, suppose that $\dot{A}$ and $\ddot{A}$ is Q-FSR and $\pi$-Q-FSR. Then we have, $\eta_{A}(a-b, q) \geqslant \min \left\{\eta_{A}(a, q), \varphi_{A}(b, q)\right\}$ and $\varphi_{A}((a-b), q) \geqslant\left\{\varphi_{A}(a, q), \varphi_{A}(b, q)\right\}, \eta_{A}(a b, q) \geqslant \min \left\{\eta_{A}(a, q), \eta_{A}(b, q)\right\}$ and $\varphi_{A}(a b, q) \geqslant$ $\min \left\{\varphi_{A}(a, q), \varphi_{A}(b, q)\right\}$. As $A$ is homogeneous, so,

$$
\begin{aligned}
\theta_{A}((a-b), q) & =\eta_{A}((a-b), q) e^{i \varphi_{A}((a-b), q)} \\
& \left.\geqslant \min \left\{\eta_{A}(a, q), \eta_{A}(b, q)\right\} e^{i \min \varphi_{A}(a, q), \varphi_{A}(b, q)}\right\} \\
& =\min \left\{\eta_{A}(a, q) e^{i \varphi_{A}(a, q)}, \eta_{A}(b, q) e^{i \varphi_{A}(b, q)}\right\} .
\end{aligned}
$$

Implies that $\theta_{A}((a-b), q) \geqslant\left\{\theta_{A}(a, q), \theta_{A}(b, q)\right\}$. Moreover,

$$
\begin{aligned}
\theta_{A}(a b, q) & =\eta_{A}(a b, q) e^{i \varphi_{A}(a b, q)} \\
& \geqslant\left\{\eta_{A}(a, q), \eta_{A}(b, q)\right\} e^{i \min \left\{\varphi_{A}(a, q), \varphi_{A}(b, q)\right\}} \\
& =\left\{\eta_{A}(a, q) e^{i \varphi_{A}(a, q)}, \eta_{A}(b, q) e^{i \varphi_{A}(b, q)}\right\} \\
& =\left\{\theta_{A}(a, q), \theta_{A}(b, q)\right\} .
\end{aligned}
$$

Hence, $A$ is Q-CFSR.

Definition 3.7. Let $A=\left\{\left(\left(a, \theta_{A}\right), q\right): q \in Q, a \in P,\right\}$ be a Q-CFS of P. For $t_{1} \in[0,1]$, and $t_{2} \in[0,2 \pi]$ the level subset of Q-CFS is defined by

$$
A_{\left(t_{1}, t_{2}\right)}=\left\{a \in P: \eta_{A}(a, q) \geqslant t_{1}, \varphi_{A}(a, q) \geqslant t_{2}\right\} .
$$

For $t_{2}=0$, we obtain the level subset $A_{t_{1}}=\left\{a \in P: \eta_{A}(a, q) \geqslant t_{1}\right\}$ and for $t_{2}=0$, then we obtain the lower level subset $A_{t_{2}}=\left\{a \in P: \varphi_{A}(a, q) \geqslant t_{2}\right\}$. 
The following result proves that level subset of Q-CFSR is sub-ring of ring.

Theorem 3.8. Let $\mathrm{A}=\left\{\left(\mathrm{a}, \theta_{\mathrm{A}}(\mathrm{a}, \mathrm{q})\right): \mathrm{a} \in \mathrm{S}, \mathrm{q} \in \mathrm{Q}\right\}$ be $\mathrm{Q}-C F S$ of ring $\mathrm{S}$. Then $\mathrm{A}$ is a $\mathrm{Q}-C F S R$ of $\mathrm{R}$ if and only if $A_{\left(t_{1}, t_{2}\right)}$ is a sub-ring of ring $S$, where $\eta_{A}(0, q) \geqslant t_{1}, \varphi_{A}(0, q) \geqslant t_{2}$ and 0 is identity element of $S$.

Proof. Obviously $A_{\left(t_{1}, t_{2}\right)}$ is nonempty, as $0 \in A_{\left(t_{1}, t_{2}\right)}$. Let $a, b \in A_{\left(t_{1}, t_{2}\right)}$ and $q \in Q$ be any two elements. Then

$$
\eta_{A}(a, q) \geqslant t_{1}, \varphi_{A}(a, q) \geqslant t_{2} \text { and } \eta_{A}(b, q) \geqslant t_{1}, \varphi_{A}(b, q) \geqslant t_{2}
$$

Consider,

$$
\begin{aligned}
\eta_{A}((a-b), q) e^{i \varphi_{A}((a-b), q)} & =\theta_{A}((a-b), q) \\
& \geqslant \min \left\{\theta_{A}(a, q), \theta_{A}(b, q)\right\} \\
& =\min \left\{\eta_{A}(a, q) e^{i \varphi_{A}(a, q)}, \eta_{A}(b, q) e^{i \varphi_{A}(b, q)}\right\} \\
& =\min \left\{\eta_{A}(a, q), \eta_{A}(b, q)\right\} e^{i \min \left\{\varphi_{A}(a, q), \varphi_{A}(b, q)\right\}} .
\end{aligned}
$$

As $A$ is homogeneous

$$
\begin{aligned}
\eta_{A}((a-b), q) & \geqslant\left\{\eta_{A}(a, q), \eta_{A}(b, q)\right\}=\left\{t_{1}, t_{1}\right\}=t_{1}, \\
\varphi_{A}((a-b), q) & \geqslant\left\{\varphi_{A}(a, q), \varphi_{A}(b, q)\right\}=\left\{t_{2}, t_{2}\right\}=t_{2}, \\
& \Rightarrow a-b \in A_{\left(t_{1}, t_{2}\right) .}
\end{aligned}
$$

Further,

$$
\begin{aligned}
\eta_{A}((a b), q) e^{i \varphi_{A}((a b), q)} & =\theta_{A}((a b), q) \\
& \geqslant \min \left\{\theta_{A}(a, q), \theta_{A}(b, q)\right. \\
& =\min \left\{\eta_{A}(a, q) e^{i \varphi_{A}(a, q)}, \eta_{A}(b, q) e^{i \varphi_{A}}(b, q)\right\} \\
& =\min \left\{\eta_{A}(a, q), \eta_{A}(b, q)\right\} e^{i \min \left\{\varphi_{A}(a, q), \varphi_{A}(b, q)\right\}}
\end{aligned}
$$

As $A$ is homogeneous

$$
\begin{aligned}
\eta_{A}((a b), q) & \geqslant\left\{\eta_{A}(a, q), \eta_{A}(b, q)\right\}=\left\{t_{1}, t_{1}\right\}=t_{1} \\
\varphi_{A}((a b), q) & \geqslant\left\{\varphi_{A}(a, q), \varphi_{A}(b, q)\right\}=\left\{t_{2}, t_{2}\right\}=t_{2}, \\
& \Rightarrow a b \in A_{\left(t_{1}, t_{2}\right)} .
\end{aligned}
$$

Hence $A_{\left(t_{1}, t_{2}\right)}$ is sub-ring. Conversely, Let $A_{\left(t_{1}, t_{2}\right)}$ is a sub-ring of $S$ and let $\min \left\{\eta_{A}(a, q), \eta_{A}(b, q)\right\}=t_{1}$ and $\min \left\{\varphi_{A}(a, q), \varphi_{A}(b, q)\right\}=t_{2}$. Then we have

$$
\begin{aligned}
& \eta_{A}(a, q) \geqslant t_{1}, \eta_{A}(b, q) \geqslant t_{1}, \varphi_{A}(a, q) \geqslant t_{2}, \text { and } \varphi_{A}(b, q) \geqslant t_{2} \\
& \eta_{A}(a, q) \geqslant t_{1}, \varphi_{A}(a, q) \geqslant t_{2}, \eta_{A}(b, q) \geqslant t_{1}, \varphi_{A}(b, q) \geqslant t_{2} .
\end{aligned}
$$

Implies that $a, b \in A_{\left(t_{1}, t_{2}\right)}$. As $A_{\left(t_{1}, t_{2}\right)}$ is subgroup

$$
\begin{aligned}
& a-b, a b \in A_{\left(t_{1}, t_{2}\right)} \Rightarrow \eta_{A}(a-b, q) \geqslant t_{1}, \varphi_{A}(a-b, q) \geqslant t_{2}, \\
& \Rightarrow \eta_{A}(a-b, q) \geqslant \min \left\{\eta_{A}(a, q), \eta_{A}(b, q)\right\} \text { and } \varphi_{A}(a-b, q) \geqslant \min \left\{\varphi_{A}(a, q), \varphi_{A}(b, q)\right\} .
\end{aligned}
$$

Implies that $\theta_{A}((a-b), q) \geqslant \min \left\{\theta_{A}(a, q), \theta_{A}(b, q)\right\}$. Moreover, $\eta_{A}(a b, q) \geqslant t_{1}, \varphi_{A}(a b, q) \geqslant t_{2}$,

$$
\Rightarrow \eta_{A}(a b, q) \geqslant \min \left\{\eta_{A}(a, q), \eta_{A}(b, q)\right\} \text { and } \varphi_{A}(a b, q) \geqslant \min \left\{\varphi_{A}(a, q), \varphi_{A}(b, q)\right\} \text {. }
$$

Implies that $\theta_{A}(a b, q) \geqslant \min \left\{\theta_{A}(a, q), \theta_{A}(b, q)\right\}$. This established the proof.

Theorem 3.9. Let $A$ be $a$ Q-CFSR of R. Then $A_{t_{1}}=\left\{a \in P\right.$ and $\left.q \in Q: \eta_{A}(a, q) \geqslant t_{1}\right\}$ and $A_{t_{2}}=\{a \in P$ : $\left.\eta_{A}(a, q) \geqslant t_{2}\right\}$ are two sub-rings of $S$. Where $\eta_{A}(0, q) \geqslant t_{1}, \varphi_{A}(0, q) \geqslant t_{2}$ and 0 is identity element of $S$.

Proof. Trivial. 


\section{Properties of the direct product of Q-complex fuzzy sub-rings}

In this section, we use the concept of Q-CFSR to define direct product of Q-CFSR. We prove that direct product of two Q-CFSR is Q-CFSR and investigate their properties.

Definition 4.1. Let $A$ and $B$ be any two $\pi$-Q-CFSs of sets $S_{1}$ and $S_{2}$, respectively. The cartesian product of $\pi$-Q-CFSs $A$ and $B$ is defined as $A_{\pi} \times B_{\pi}((m, n), q)=\min \left\{A_{\pi}(m, q), B_{\pi}(n, q)\right\}$, for all $m \in S_{1}$ and $n \in$ $S_{2}$ and $\mathrm{q} \in \mathrm{Q}$.

Remark 4.2. Let $A$ and $B$ be two $\pi$-Q-CFSR of $S_{1}$ and $S_{2}$, respectively. Then $A \times B$ is $\pi$-Q-FSG of $S_{1} \times S_{2}$.

Definition 4.3. Let $A$ and B two Q-CFSR of sets $S_{1}$ and $S_{2}$. The cartesian product of Q-CFSRs $A$ and B is defined by a function

$$
\theta_{A \times B}((m, n), q)=\eta_{A \times B}((m, n), q) e^{i \varphi_{A \times B}((m, n), q)}=\min \left\{\eta_{A}(m, q), \eta_{B}(n, q)\right\} e^{i \min \left\{\varphi_{A}(m, q), \varphi_{B}(n, q)\right\}} .
$$

The following results depicted that the direct product of two Q-CFSR is also Q-CFSR.

Theorem 4.4. Let $\mathrm{A}$ and $\mathrm{B}$ be two $\mathrm{Q}-$ CFSRs of $\mathrm{S}_{1}$ and $\mathrm{S}_{2}$, respectively. Then $\mathrm{A} \times \mathrm{B}$ is $\mathrm{Q}-C F S R$ of $\mathrm{S}_{1} \times \mathrm{S}_{2}$.

Proof. Let $m, x \in S_{1}$ and $n, y \in S_{2}$ be elements and $q \in Q$. Then $(m, n),(x, y) \in S_{1} \times S_{2}$. Consider,

$$
\begin{aligned}
\theta_{A \times B}((m, n)-(x, y), q) & =\theta_{A \times B}((m-x, n-y), q) \\
& =\eta_{A \times B}((m-x, n-y), q) e^{i \varphi_{A \times B}((m-x, n-y), q)} \\
& =\min \left\{\eta_{A}(m-x, q), \eta_{B}(n-y, q)\right\} e^{i \min \left\{\varphi_{A}(m-x, q), \varphi_{B}(n-y, q)\right\}} \\
& =\min \left\{\eta_{A}(m-x, q) e^{i \varphi_{A}(m-x, q)}, \eta_{B}(n-y, q) e^{i \varphi_{A}(n-y, q)}\right\} \\
& =\min \left\{\theta_{A}(m-x, q), \theta_{B}(n-y, q)\right\} \\
& \geqslant \min \left\{\min \left\{\theta_{A}(m, q), \theta_{A}(x, q)\right\}, \min \left\{\theta_{B}(n, q), \theta_{B}(y, q)\right\}\right\} \\
& =\min \left\{\min \left\{\theta_{A}(m, q), \theta_{B}(n, q)\right\}, \min \left\{\theta_{A}(x, q), \theta_{B}(y, q)\right\}\right\} .
\end{aligned}
$$

Thus, $\theta_{A \times B}((m, n)-(x, y), q) \geqslant \min \left\{\theta_{A \times B}((m, n), q), \theta_{A \times B}((x, y), q)\right\}$.

Further,

$$
\begin{aligned}
\theta_{A \times B}((m, n)(x, y), q) & =\theta_{A \times B}((m x, n y), q) \\
& =\eta_{A \times B}((m x, n y), q) e^{i \varphi_{A \times B}((m x, n y), q)} \\
& =\min \left\{\eta_{A}(m x, q), \eta_{B}(n y, q)\right\} e^{i \min \left\{\varphi_{A}(m x, q), \varphi_{B}(n y, q)\right\}} \\
& =\min \left\{\eta_{A}(m x, q) e^{i \varphi_{A}(m x, q)}, \eta_{B}(n y, q) e^{i \varphi_{A}(n y, q)}\right\} \\
& =\min \left\{\theta_{A}(m x, q), \theta_{B}(n y, q)\right\} \\
& \geqslant \min \left\{\min \left\{\theta_{A}(m, q), \theta_{A}(x, q)\right\}, \min \left\{\theta_{B}(n, q), \theta_{B}(y, q)\right\}\right\} \\
& =\min \left\{\min \left\{\theta_{A}(m, q), \theta_{B}(n, q)\right\}, \min \left\{\theta_{A}(x, q), \theta_{B}(y, q)\right\}\right\} .
\end{aligned}
$$

Therefore, $\theta_{A \times B}((m, n)(x, y), q) \geqslant \min \left\{\theta_{A \times B}((m, n), q), \theta_{A \times B}((x, y), q)\right\}$. This concludes the proof.

Corollary 4.5. Let $A_{1}, A_{2}, \ldots, A_{n}$ be Q-CFSRs of $S_{1}, S_{2}, \ldots, S_{n}$, respectively. Then $A_{1} \times A_{2} \times \cdots \times A_{n}$ is QCFSR of $S_{1} \times S_{2} \times \cdots \times S_{n}$.

Remark 4.6. Let $A_{1}$ and $A_{2}$ be two Q-CFSRs of $S_{1}$ and $S_{2}$, respectively and $A_{1} \times A_{2}$ be Q-CFSR of $S_{1} \times S_{2}$. Then it is not compulsory both $A_{1}$ and $A_{2}$ should be Q-CFSR of $S_{1}$ and $S_{2}$, respectively. 
Example 4.7. Let $\bar{Z}_{2}=\{0,1\}$ and $S=\{e, a, b, c\}$ be two rings. Here $S$ is ring of $2 \times 2$ matrices over $\bar{Z}_{2}$ with second row has zero entries, where $e=\left[\begin{array}{ll}0 & 0 \\ 0 & 0\end{array}\right], a=\left[\begin{array}{ll}0 & 1 \\ 0 & 0\end{array}\right], b=\left[\begin{array}{ll}1 & 0 \\ 0 & 0\end{array}\right], c=\left[\begin{array}{ll}1 & 1 \\ 0 & 0\end{array}\right]$.

$\bar{Z}_{2} \times S=\{(0, e),(0, a),(0, b),(0, c),(1, e),(1, a),(1, b),(1, c)\}$. Then two Q-CFSRs $A_{1}$ and $A_{2}$ of $\bar{Z}_{2}$ and $S$ are defined by

$$
\begin{aligned}
A_{1} & =\left\{\left((0, q), 0.3 e^{i \frac{\pi}{12}}\right),\left((1, q), 0.2 e^{i \frac{\pi}{15}}\right)\right\}, \text { where } q \in Q, \\
A_{2} & =\left\{\left((e, q), 0.4 e^{i \frac{\pi}{3}}\right),\left((a, q), 0.55 e^{i \frac{\pi}{2}}\right),\left(\left(a^{2}, q\right), 0.43 e^{i \frac{\pi}{3}}\right),\left(\left(a^{3}, q\right), 0.5 e^{i \pi}\right)\right\}, \\
\left(A_{1} \times A_{2}\right)(m, q) & = \begin{cases}0.3 e^{i \frac{\pi}{12}}, & \text { for all } m \in\{(0, e),(0, a),(0, b),(0, c)\}, \\
0.2 e^{i \frac{\pi}{15}}, & \text { for all } m \in\{(1, e),(1, a),(1, b),(1, c)\} .\end{cases}
\end{aligned}
$$

Here, $A_{1} \times A_{2}$ is CFSG of $Z_{2} \times S$ and $A_{1}$ is Q-CFSR of $Z_{2}$. But $A_{2}$ is not a Q-CFSR of $S$ of $H_{1}$. But $A_{2}$ is not a Q-CFSR of $S_{2}$.

Theorem 4.8. Let $\mathrm{A}$ and $\mathrm{B}$ be two $\mathrm{Q}-C F S$ s of rings $\mathrm{S}_{1}$ and $\mathrm{S}_{2}$, respectively. If $\mathrm{A} \times \mathrm{B}$ is a $\mathrm{Q}-C F S R$ of $\mathrm{S}_{1} \times \mathrm{S}_{2}$, then at least one of the following statements must be hold.

1. $\eta_{A}(0, q) \geqslant \eta_{B}(n, q)$ and $\varphi_{A}(0, q) \geqslant \varphi_{B}(n, q)$, for all $n \in S_{2}$ and $q \in Q$;

2. $\eta_{B}\left(0^{\prime}, q\right) \geqslant \eta_{A}(m, q)$ and $\varphi_{B}\left(0^{\prime}\right) \geqslant \varphi_{A}(m)$, for all $m \in S_{1}$ and $q \in Q$.

Where 0 and $0^{\prime}$ are identities of $S_{1}$ and $S_{2}$, respectively.

Proof. Let $A \times B$ be a Q-CFSR of $S_{1} \times S_{2}$. On contrary, suppose that the statements 1 and 2 do not hold. Then there exist $m \in S_{1}$ and $n \in S_{2}$ and $q \in Q$ such that

1. $\eta_{A}(0, q) \leqslant \eta_{B}(n, q)$ and $\varphi_{A}(0, q) \leqslant \varphi_{B}(n, q)$;

2. $\eta_{B}\left(0^{\prime}, q\right) \leqslant \eta_{A}(m, q)$ and $\varphi_{B}\left(0^{\prime}, q\right) \leqslant \varphi_{A}(m, q)$.

Consider

$$
\begin{aligned}
\theta_{A \times B}((m, n), q) & =\min \left\{\eta_{A}(m, q), \eta_{B}(n, q)\right\} e^{i \min \left\{\varphi_{A}(m, q), \varphi_{B}(n, q)\right\}} \\
& \geqslant \min \left\{\eta_{A}(0, q), \eta_{B}\left(0^{\prime}, q\right)\right\} e^{i \min \left\{\varphi_{A}(0, q), \varphi_{B}\left(0^{\prime}, q\right)\right\}}=\theta_{A \times B}\left(\left(0,0^{\prime}\right), q\right) .
\end{aligned}
$$

But $A \times B$ is $Q-C F S R$. Hence, at least one of the following statements must hold,

1. $\eta_{A}(0, q) \geqslant \eta_{B}(n, q)$ and $\varphi_{A}(0, q) \geqslant \varphi_{B}(n, q)$, for all $n \in S_{2}$ and $q \in Q$;

2. $\eta_{B}\left(0^{\prime}, q\right) \geqslant \eta_{A}(m, q)$ and $\varphi_{B}\left(0^{\prime}, q\right) \geqslant \varphi_{A}(m, q)$, for all $m \in S_{1}$ and $q \in Q$.

Theorem 4.9. Let $A$ and $B$ Q-CFSRs of $S_{1}$ and $S_{2}$ such that $\eta_{B}\left(0^{\prime}, q\right) \geqslant \eta_{A}(m, q)$ and $\varphi_{B}\left(0^{\prime}, q\right) \geqslant \varphi_{A}(m, q)$ for all $\mathrm{m} \in \mathrm{S}_{1}$ and $0^{\prime}$ is identity of $\mathrm{S}_{2}$ and $\mathrm{q} \in \mathrm{Q}$. If $\mathrm{A} \times \mathrm{B}$ is $\mathrm{Q}-C F S R$ of $\mathrm{S}_{1} \times \mathrm{S}_{2}$, then $\mathrm{A}$ is $\mathrm{Q}-C F S R$ of $\mathrm{S}_{1}$.

Proof. Let $A$ and $B$ be two Q-CFSRs of $S_{1}$ and $S_{2}$. Then $\left(m, 0^{\prime}\right),\left(x, 0^{\prime}\right) \in S_{1} \times S_{2}$. By given condition $\eta_{B}\left(0^{\prime}, q\right) \geqslant \eta_{A}(m, q)$ and $\varphi_{B}\left(0^{\prime}, q\right) \geqslant \varphi_{A}(m, q)$, for all $m, x \in S_{1}$ we have

$$
\begin{aligned}
& \theta_{A}(m-x, q) \\
& =\eta_{A}(m-x, q) e^{i \varphi_{A}(m-x, q)} \\
& =\min \left\{\eta_{A}(m-x, q) e^{i \varphi_{A}(m-x, q)}, \eta_{B}\left(0^{\prime}-0^{\prime}, q\right) e^{i \varphi_{B}\left(0^{\prime}-0^{\prime}, q\right)}\right\} \\
& =\left\{\eta_{A \times B}\left(\left(m, 0^{\prime}\right)-\left(x, 0^{\prime}\right), q\right)\right\} e^{i \min \left\{\varphi_{A \times B}\left(\left(m, 0^{\prime}\right)-\left(x, 0^{\prime}\right), q\right)\right\}} \\
& \geqslant \min \left\{\eta_{A \times B}\left(\left(m, 0^{\prime}\right), q\right), \eta_{A \times B}\left(\left(x, 0^{\prime}\right), q\right)\right\} e^{i \min \left\{\varphi_{A \times B}\left(\left(m, 0^{\prime}\right), q\right), \varphi_{A \times B}\left(\left(x, 0^{\prime}\right), q\right)\right\}} \\
& =\min \left\{\min \left\{\eta_{A}(m, q), \eta_{B}\left(0^{\prime}, q\right)\right\}, \min \left\{\eta_{A}(x, q), \eta_{B}\left(0^{\prime}, q\right)\right\}\right\} e^{i \operatorname{imin}\left\{\min \left\{\varphi_{A}(m, q), \varphi_{B}\left(0^{\prime}, q\right)\right\}, \min \left\{\varphi_{A}(x, q), \varphi_{B}\left(0^{\prime}, q\right)\right\}\right\}}
\end{aligned}
$$




$$
\begin{aligned}
& =\min \left\{\min \left\{\eta_{A}(m, q), \eta_{A}(m, q)\right\}, \min \left\{\eta_{A}(x, q), \eta_{A}(x, q)\right\}\right\} e^{i \operatorname{inin}\left\{\min \left\{\eta_{A}(m, q), \eta_{A}(m, q)\right\}, \min \left\{\eta_{A}(x, q), \eta_{A}(x, q)\right\}\right\}} \\
& =\min \left\{\eta_{A}(m, q), \eta_{A}(x, q)\right\} e^{i \min \left\{\varphi_{A}(m, q), \varphi_{A}(x, q)\right\}} \\
& =\min \left\{\theta_{A}(m, q), \theta_{A}(x, q)\right\} .
\end{aligned}
$$

Thus, $\theta_{A}(m-x, q) \geqslant \min \left\{\theta_{A}(m, q), \theta_{A}(x, q)\right\}$. Also,

$$
\begin{aligned}
& \theta_{A}(m x, q) \\
& =\eta_{A}(m x, q) e^{i \varphi_{A}(m x, q)} \\
& =\min \left\{\eta_{A}(m x, q) e^{i \varphi_{A}(m x, q)}, \eta_{B}\left(0^{\prime} 0^{\prime}, q\right) e^{i \varphi_{B}\left(0^{\prime} 0^{\prime}, q\right)}\right\} \\
& =\left\{\eta_{A \times B}\left(\left(m, 0^{\prime}\right)\left(x, 0^{\prime}\right), q\right)\right\} e^{i \min \left\{\varphi_{A \times B}\left(\left(m, 0^{\prime}\right)\left(x, 0^{\prime}\right), q\right)\right\}} \\
& \geqslant \min \left\{\eta_{A \times B}\left(\left(m, 0^{\prime}\right), q\right), \eta_{A \times B}\left(\left(x, 0^{\prime}\right), q\right)\right\} e^{i \min \left\{\varphi_{A \times B}\left(\left(m, 0^{\prime}\right), q\right), \varphi_{A \times B}\left(\left(x, 0^{\prime}\right), q\right)\right\}} \\
& =\min \left\{\min \left\{\eta_{A}(m, q), \eta_{B}\left(0^{\prime}, q\right)\right\}, \min \left\{\eta_{A}(x, q), \eta_{B}\left(0^{\prime}, q\right)\right\}\right\} e^{i \min \left\{\min \left\{\varphi_{A}(m, q), \varphi_{B}\left(0^{\prime}, q\right)\right\}, \min \left\{\varphi_{A}(x, q), \varphi_{B}\left(0^{\prime}, q\right)\right\}\right\}} \\
& =\min \left\{\min \left\{\eta_{A}(m, q), \eta_{A}(m, q)\right\}, \min \left\{\eta_{A}(x, q), \eta_{A}(x, q)\right\}\right\} e^{i \min \left\{\min \left\{\eta_{A}(m, q), \eta_{A}(m, q)\right\}, \min \left\{\eta_{A}(x, q), \eta_{A}(x, q)\right\}\right\}} \\
& =\min \left\{\eta_{A}(m, q), \eta_{A}(x, q)\right\} e^{i \min \left\{\varphi_{A}(m, q), \varphi_{A}(x, q)\right\}} \\
& =\min \left\{\theta_{A}(m, q), \theta_{A}(x, q)\right\}
\end{aligned}
$$

Thus, $\theta_{A}(m x, q) \geqslant \min \left\{\theta_{A}(m, q), \theta_{A}(x, q)\right\}$. Hence, proved our claim.

Theorem 4.10. Let $A$ and $B$ two Q-CFSRs of $S_{1}$ and $S_{2}$ such that $\eta_{A}(0, q) \geqslant \eta_{B}(n, q)$ and $\varphi_{A}(0, q) \geqslant \varphi_{B}(n, q)$ for all $\mathrm{n} \in \mathrm{S}_{2}$ and 0 is identity of $\mathrm{S}_{1}$ and $\mathrm{q} \in \mathrm{Q}$. If $\mathrm{A} \times \mathrm{B}$ is $\mathrm{Q}-C F S R$ of $\mathrm{S}_{1} \times \mathrm{S}_{2}$, then $\mathrm{B}$ is $\mathrm{Q}-C F S R$ of $\mathrm{S}_{2}$.

Proof. Similar as pervious theorem.

Corollary 4.11. Let $\mathrm{A}$ and $\mathrm{B}$ two Q-CFSRs of $\mathrm{S}_{1}$ and $\mathrm{S}_{2}$ respectively. If $\mathrm{A} \times \mathrm{B}$ is $\mathrm{Q}-C F S R$ of $\mathrm{S}_{1} \times \mathrm{S}_{2}$ and then $\mathrm{A}$ is a $\mathrm{Q}-C F S R$ of $\mathrm{S}_{1}$ or $\mathrm{B}$ is a $\mathrm{Q}-C F S R$ of $\mathrm{S}_{2}$.

\section{Homomorphism of Q-complex fuzzy sub-rings}

In this section, we define the homomorphic image and pre image of the Q-CFSR. We prove some results of the Q-CFSR under ring homomorphism.

Definition 5.1. Let $f: S \rightarrow R$ be a homomorphism from $\operatorname{ring} S$ to $\operatorname{ring} R$. Let $A$ and $B$ be two Q-CFSRs of rings $S$ and $R$, respectively. For all $m \in S$ and for all $n \in R$, the image $f(A)$ and pre-image $f^{-1}(B)$ of $A$ and $B$, respectively, are defined as

$$
f\left(\theta_{A}\right)(n, q)=\left\{\begin{array}{ll}
\sup \left\{\theta_{A}(m, q),\right. & \text { if } f(m)=n, f^{-1}(n) \neq \emptyset, \\
1, & \text { otherwise, }
\end{array} \quad f^{-1}\left(\theta_{B}\right)(m, q)=\theta_{B}(f(m, q)) .\right.
$$

Theorem 5.2. Let $\mathrm{f}: \mathrm{S} \rightarrow \mathrm{R}$ be homomorphism from ring $\mathrm{S}$ to ring $\mathrm{R}$. Let $\mathrm{A}$ be $\mathrm{Q}-C F S R$ of $\mathrm{S}$ and $\mathrm{B}$ be $\mathrm{Q}-C F S R$ of $\mathrm{R}$. Then $\mathrm{f}(\mathrm{A})$ is $\mathrm{Q}-$ CFSR of $\mathrm{R}$ and $\mathrm{f}^{-1}(\mathrm{~B})$ is $\mathrm{Q}-$ CFSR of $\mathrm{S}$.

Lemma 5.3. Let $\mathrm{f}: \mathrm{S} \rightarrow \mathrm{R}$ be a ring homomorphism from $\mathrm{S}$ to $\mathrm{R}$. Let $\mathrm{A}$ and $\mathrm{B}$ be two $\mathrm{Q}-$ CFSRs. Then

1. $f\left(\theta_{A}\right)(n, q)=f\left(\eta_{A}\right)(n, q) e^{i f\left(\varphi_{A}\right)(n, q)}$, for all $m \in S$ and $q \in Q$;

2. $f^{-1}\left(\theta_{B}\right)(m, q)=f^{-1}\left(\eta_{B}\right)(m, q) e^{i f^{-1}\left(\varphi_{B}\right)(m, q)}$, for all $n \in R$ and $q \in Q$.

Proof. Consider,

$$
f\left(\theta_{A}\right)(n, q)=\max \left\{\left(\theta_{A}\right)(m, q), \text { if } f(m)=n\right\}
$$




$$
\begin{aligned}
& =\max \left\{\left(\eta_{A}\right)(m, q) e^{i f\left(\varphi_{A}\right)(m, q)}, \quad \text { if } f(m)=(n)\right\} \\
& =\max \left\{\left(\eta_{A}\right)(m, q), \quad \text { if } f(m)=n\right\} e^{i \max \left\{\left(\varphi_{A}\right)(m, q), \quad \text { if } f(m)=n\right\}} \\
& =f\left(\eta_{A}\right)(n, q) e^{i f\left(\varphi_{A}\right)(n, q)} .
\end{aligned}
$$

Hence, $f\left(\theta_{A}\right)(n, q)=f\left(\eta_{A}\right)(n, q) e^{i f\left(\varphi_{A}\right)(n, q)}$.

Consider,

$$
f^{-1}\left(\theta_{B}\right)(m, q)=\theta_{B}(f(m, q))=\eta_{B}(f(m, q)) e^{i \varphi_{B}(f(m, q))}=f^{-1}\left(\eta_{B}\right)(m, q) e^{i f^{-1}\left(\varphi_{B}\right)(m, q)} .
$$

The following result leads us to prove that homomorphic image of Q-CFSR is also Q-CFSR.

Theorem 5.4. Let $\mathrm{f}: \mathrm{S} \rightarrow \mathrm{R}$ be a ring homomorphism from $\mathrm{S}$ to $\mathrm{R}$. Let $\mathrm{A}$ be $\mathrm{Q}-C F S R$ of $\mathrm{S}$. Then $\mathrm{f}(\mathrm{A})$ is $\mathrm{Q}-C F S R$ of $\mathrm{R}$.

Proof. Obviously, $\eta_{A}$ and $\varphi_{A}$ are Q-FSR and $\pi$-Q-FSR, respectively. From theorem 3.3, 3.6 and theorem 5.1 the homomorphic image of $\eta_{A}$ and $\varphi_{A}$ are Q-FSR and $\pi$-Q-FSR respectively, for all $m, n \in R$. Then we have

$$
\begin{gathered}
f\left(\eta_{B}\right)(m-n, q) \geqslant \min \left\{f\left(\eta_{B}\right)(m, q), f\left(\eta_{B}\right)(n, q)\right\} \\
f\left(\eta_{B}\right)(m n, q) \geqslant \min \left\{f\left(\eta_{B}\right)(m, q), f\left(\eta_{B}\right)(n, q)\right\} \\
f\left(\varphi_{B}\right)(m-n, q) \geqslant \min \left\{f\left(\varphi_{B}\right)(m, q), f\left(\varphi_{B}\right)(n, q)\right\} \\
f\left(\varphi_{B}\right)(m n, q) \geqslant \min \left\{f\left(\varphi_{B}\right)(m, q), f\left(\varphi_{B}\right)(n, q)\right\} .
\end{gathered}
$$

Consider,

$$
\begin{aligned}
f\left(\theta_{A}\right)(m-n, q) & =f\left(\eta_{A}\right)(m-n, q) e^{i f\left(\varphi_{A}\right)(m-n, q)}, \quad \forall m, n \in R \\
& \geqslant \min \left\{f\left(\eta_{A}\right)(m, q), f\left(\eta_{A}\right)(n, q)\right\} e^{i\left\{f\left(\varphi_{A}\right)(m, q), f\left(\varphi_{A}\right)(n, q)\right\}} \\
& \geqslant \min \left\{f\left(\eta_{A}\right)(m, q) e^{i f\left(\varphi_{A}\right)(m, q)}, f\left(\eta_{A}\right)(n, q) e^{i f\left(\varphi_{A}\right)(n, q)}\right\} \\
& =\min \left\{f\left(\theta_{A}\right)(m, q), f\left(\theta_{A}\right)(n, q)\right\} .
\end{aligned}
$$

Consequently, $f\left(\theta_{A}\right)(m-n, q) \geqslant \min \left\{f\left(\theta_{A}\right)(m, q), f\left(\theta_{A}\right)(n, q)\right\}$.

Further,

$$
\begin{aligned}
f\left(\theta_{A}\right)(m n, q) & =\left(\eta_{A}\right)(m n, q) e^{i f\left(\varphi_{A}\right)(m n, q)}, \quad \forall m, n \in R \\
& \geqslant \min \left\{f\left(\eta_{A}\right)(m, q), f\left(\eta_{A}\right)(n, q)\right\} e^{i\left\{f\left(\varphi_{A}\right)(m, q), f\left(\varphi_{A}\right)(n, q)\right\}} \\
& \geqslant \min \left\{f\left(\eta_{A}\right)(m, q) e^{i f\left(\varphi_{A}\right)(m, q)}, f\left(\eta_{A}\right)(n, q) e^{i f\left(\varphi_{A}\right)(n, q)}\right\} \\
& =\min \left\{f\left(\theta_{A}\right)(m, q), f\left(\theta_{A}\right)(n, q)\right\} .
\end{aligned}
$$

Consequently, $f\left(\theta_{A}\right)(m n, q) \geqslant \min \left\{f\left(\theta_{A}\right)(m, q), f\left(\theta_{A}\right)(n, q)\right\}$. This establishes the proof.

Theorem 5.5. Let $\mathrm{f}: \mathrm{S} \rightarrow \mathrm{R}$ be a homomorphism from ring $\mathrm{S}$ to ring $\mathrm{R}$. Let $\mathrm{B}$ be two $\mathrm{Q}-C F S R$ of $\mathrm{R}$. Then $\mathrm{f}^{-1}(\mathrm{~B})$ is Q-CFSR of S.

Proof. Obviously, $\eta_{\mathrm{B}}$ and $\varphi_{\mathrm{B}}$ are Q-FSR and $\pi$-Q-FSR respectively. Then From Theorems 3.3, 3.6, and 5.2, the inverse image of $\eta_{B}$ and $\varphi_{B}$ are Q-FSR and $\pi$-Q-FSR, respectively, for all $m, n \in S$. Then we have

$$
\begin{aligned}
f^{-1}\left(\eta_{B}\right)(m-n, q) & \geqslant \min \left\{f^{-1}\left(\eta_{B}\right)(m, q), f^{-1}\left(\eta_{B}\right)(n, q)\right\}, \\
f^{-1}\left(\eta_{B}\right)(m n, q) & \geqslant \min \left\{f^{-1}\left(\eta_{B}\right)(m, q), f^{-1}\left(\eta_{B}\right)(n, q)\right\}, \\
f^{-1}\left(\varphi_{B}\right)(m-n, q) & \geqslant \min \left\{f^{-1}\left(\varphi_{B}\right)(m, q), f^{-1}\left(\varphi_{B}\right)(n, q)\right\}, \\
f^{-1}\left(\varphi_{B}\right)(m n, q) & \geqslant \min \left\{f^{-1}\left(\varphi_{B}\right)(m, q), f^{-1}\left(\varphi_{B}\right)(n, q)\right\} .
\end{aligned}
$$


Consider,

$$
\begin{aligned}
f^{-1}\left(\theta_{B}\right)(m-n, q) & =f^{-1}\left(\eta_{B}\right)(m-n, q) e^{i f^{-1}\left(\varphi_{B}\right)(m-n, q)}, \text { for all } m, n \in S \text { and } q \in Q \\
& \geqslant \min \left\{f^{-1}\left(\eta_{B}\right)(m, q), f^{-1}\left(\eta_{B}\right)(n, q)\right\} e^{i\left\{f^{-1}\left(\varphi_{B}\right)(m, q), f^{-1}\left(\varphi_{B}\right)(n, q)\right\}} \\
& \geqslant \min \left\{f^{-1}\left(\eta_{B}\right)(m, q) e^{i f^{-1}\left(\varphi_{B}\right)(m, q)}, f^{-1}\left(\eta_{B}\right)(n, q) e^{i f^{-1}\left(\varphi_{B}\right)(n, q)}\right\} \\
& =\min \left\{f^{-1}\left(\theta_{B}\right)(m, q), f^{-1}\left(\theta_{B}\right)(n, q)\right\} .
\end{aligned}
$$

Therefore, $f^{-1}\left(\theta_{B}\right)(m-n, q) \geqslant \min \left\{f^{-1}\left(\theta_{B}\right)(m, q), f^{-1}\left(\theta_{B}\right)(n, q)\right\}$. Further,

$$
\begin{aligned}
f^{-1}\left(\theta_{B}\right)(m n, q) & =f^{-1}\left(\eta_{B}\right)(m n, q) e^{i f^{-1}\left(\varphi_{B}\right)(m n, q)}, \text { for all } m, n \in S \text { and } q \in Q \\
& \geqslant \min \left\{f^{-1}\left(\eta_{B}\right)(m, q), f^{-1}\left(\eta_{B}\right)(n, q)\right\} e^{i\left\{f^{-1}\left(\varphi_{B}\right)(m, q), f^{-1}\left(\varphi_{B}\right)(n, q)\right\}} \\
& \geqslant \min \left\{f^{-1}\left(\eta_{B}\right)(m, q) e^{i f^{-1}\left(\varphi_{B}\right)(m, q)}, f^{-1}\left(\eta_{B}\right)(n, q) e^{i f^{-1}\left(\varphi_{B}\right)(n, q)}\right\} \\
& \geqslant \min \left\{f^{-1}\left(\theta_{B}\right)(m, q), f^{-1}\left(\theta_{B}\right)(n, q)\right\} .
\end{aligned}
$$

Consequently, $f^{-1}\left(\theta_{B}\right)(m n, q) \geqslant \min \left\{f^{-1}\left(\theta_{B}\right)(m, q), f^{-1}\left(\theta_{B}\right)(n, q)\right\}$. This concludes the proof.

\section{Conclusion}

Our purpose is to influence the research of ring algebraic structure and investigated new methodological development on ring theory, which will be useful in future. In this study, we have introduced the $\pi$-Q-FSR, Q-CFSR and level subset of Q-CFSR and have proved that level subset of Q-CFSR is sub-ring of ring S. We have also defined product of two Q-CFSRs and have proved that the product of two Q-CFSRs is also Q-CFSR and discussed various algebraic properties. Further, we have studied the behavior of homomorphic image and inverse image of these Q-CFSRs.

\section{Acknowledgment}

This work was funded by the Deanship of Scientific Research (DSR) at King Abdulaziz University, Jeddah, Saudi Arabia. The authors, therefore, acknowledge with thanks DSR for technical and financial support.

\section{References}

[1] G. M. Addis, Fuzzy homomorphism theorem on groups, Korean J. Math., 26 (2018), 373-385. 1

[2] M. N. Alemu, Fuzzy model for chaotic time series prediction, Int. J. Innov. Comput. Info. Control, 14 (2018), $1767-1786$. 1

[3] F. A. Azam, A. A. Mamun, F. Nasrin, Anti fuzzy ideal of ring, Ann. Fuzzy Math. Inform., 25 (2013), 349-360. 1

[4] J. J. Buckley, Fuzzy complex numbers, Fuzzy sets and system, 33 (1989), 333-345. 1

[5] D.-G. Chen, S.-Y. Li, Fuzzy Factor Rings, Fuzzy Sets and Systems, 94 (1998), 125-127. 1

[6] M. Gulistan, N. Yaqoob, S. Nawaz, M. Azhar, A study of $(\alpha, \beta)$-complex fuzzy hyper ideas in non-associative hyperrings, J. Intell. Fuzzy Syst., 36 (2019), 1-17. 1

[7] B. Hu, L. Bi, S. Dai, S. Li, Distances of complex fuzzy sets and continuity of complex fuzzy operations, J. Intell. Fuzzy Syst., 35 (2018), 2247-2255. 1

[8] B. Hu, L. Bi, S. Dai, S. Li, The approximate parallelity of complex fuzzy sets, J. Intell. Fuzzy Syst., 35 (2018), $6343-6351$. 1

[9] N. Kausar, Direct product of finite intuitionistic fuzzy normal subrings over non-associative rings, Eur. J. Pure Appl. Math., 12 (2019), 622-648. 1

[10] N. Kausar, B. U. Islam, M. Y. Javid, S. A. Ahmed, U. Ijaz, Characterization of non-associative rings by the properties of their fuzzy ideals, J. Taibah Univ. Sci., 13 (2019), 820-833. 1

[11] R. Kellil, Sum and product of fuzzy ideals of ring, Int. J. Comput. Sci., 33 (2018), 187-205. 1

[12] K. C. Lee, K. Hur, P. K. Lim, Interval-Valued Fuzzy Ideals of a Ring, Int. J. Fuzzy Log. Intell. Syst., 12 (2012), 198-204. 1 
[13] W. J. Liu, Fuzzy invariant subgroups and fuzzy ideals, Fuzzy sets and systems, 8 (1982), 133-139. 1, 2.2

[14] B. B. Makamba, V. Murali, A class of fuzzy subgroups of finite reflection groups, J. Intell. Fuzzy Syst., 33 (2017), 979-983. 1

[15] D. S. Malik, J. N. Mordeson, Fuzzy homomorphism of rings, Fuzzy Sets and Systems, 8 (1992), 139-146. 1

[16] Y. Qin, Y. Liu, Z. Hong, H. Jia, Hesitant fuzzy muirhead mean operators and its application to multiple attribute decision making, Int. J. Innov. Comput. Inf. Control, 14 (2018), 1223-1238. 1

[17] D. Ramot, M. Friedman, G. Langholz, A. Kandel, Complex fuzzy logic, IEEE Trans. Fuzzy Syst., 11 (2003), 171-186. 1

[18] D. Ramot, R. Milo, M. Friedman, A. Kandel, Complex fuzzy sets, IEEE Trans. Fuzzy Syst., 10 (2002), 450-461. 1, 2.5

[19] R. Rasuli, Characterization of Q-fuzzy subrings (Anti Q-fuzzy subrings) with respect to a T-norm (T-conform), J. Inf. Optimiz. Sci., 39 (2018), 827-837. 1, 2.4, 2.7

[20] A. Rosenfeld, Fuzzy groups, J. Math. Anal. Appl., 35 (1971), 512-517. 1

[21] A. Sehgal, S. Sehgal, P. K. Sharma, The number of fuzzy subgroups of a finite dihedral $\mathrm{D}_{\mathrm{pmpn}}$, Int. J. Fuzzy Math. Arch., 8 (2015), 51-57. 1

[22] A. Sehgal, S. Sehgal, P. K. Sharma, The number of fuzzy subgroups of finite abelian p-group $Z_{p m} \times Z_{p n}, A d v$. Fuzzy Sets Syst., 21 (2016), 49-57. 1

[23] G. Selvachandran, P. K. Maji, I. E. Abed, A. R. Salleh, Relations between complex vague soft sets, Appl. Soft Comput., 47 (2016), 438-448. 1

[24] D. Shan, W. Lu, J. Yang, The data-driven fuzzy cognitive map model and its application to prediction of time series, Int. J. Innov. Comput. Inf. Control, 14 (2018), 1583-1602. 1

[25] D. Singh, V. Joshi, M. Imdad, P. Kumam, A novel framework of complex valued fuzzy metric spaces and fixed point theorems, J. Intell. Fuzzy Syst., 30 (2016), 3227-3238. 1

[26] A. Solairaju, R. Nagarajan, A new structure and construction of Q-fuzzy groups, Adv. Fuzzy Math., 4 (2009), $23-29$. $1,2.3$

[27] P. Thirunavukarasu, R. Suresh, P. Thamilmani, Application of complex fuzzy sets, JP J. Appl. Math., 6 (2013), 5-22. 1

[28] P. Thirunavukarasu, R. Suresh, K. K. Viswanathan, Energy of complex fuzzy graph, Int. J. Math. Sci. Eng. Appls., 10 (2016), 243-248.

[29] S. A. Trevijano, M. J. Chasco, J. Elorza, The annihilator of fuzzy subgroups, Fuzzy Sets and Systems, 369 (2019), 122-131. 1

[30] E. Yetkin, N. Olgun, Direct product of fuzzy groups and fuzzy rings, Int. Math. Forum, 6 (2011), 1005-1015. 1

[31] Y. B. Yun, X. L. Xin, Complex fuzzy sets with application in BCK/BCI-Algebras, Bull. Sect. Log., 48 (2019), 173-185. 1

[32] L. A. Zadeh, Fuzzy set, Information and Control, 8 (1965), 338-353. 1, 2.1

[33] G. Q. Zhang, T. S. Dillon, K. Y. Cai, J. Ma, J. Lu, Operation properties and Sup-equalities of complex fuzzy sets, Internat. J. Approx. Reason, 50 (2009), 1227-1249. 1, 2.6 